BNL-112622-2016-JA

File \# 93554

\title{
Nm-scale spatial resolution $x-r a y$ imaging with MLL nanofocusing optics: instrumentational requirements and challenges
}

E. Nazaretski, H. Yan K. Lauer, X. Huang, W. Xu, S. Kalbfleisch, Hui Yan, Li Li, N. Bouet, J. Zhou, D. Shu, R. Conley, and Y. S. Chu

Submitted to AIP Conference Proceedings 1764

August 2016

Photon Sciences Department

Brookhaven National Laboratory

\author{
U.S. Department of Energy \\ USDOE Office of Science (SC), \\ Basic Energy Sciences (BES) (SC-22)
}

Notice: This manuscript has been authored by employees of Brookhaven Science Associates, LLC under Contract No. DE- SC0012704 with the U.S. Department of Energy. The publisher by accepting the manuscript for publication acknowledges that the United States Government retains a non-exclusive, paid-up, irrevocable, world-wide license to publish or reproduce the published form of this manuscript, or allow others to do so, for United States Government purposes. 


\section{DISCLAIMER}

This report was prepared as an account of work sponsored by an agency of the United States Government. Neither the United States Government nor any agency thereof, nor any of their employees, nor any of their contractors, subcontractors, or their employees, makes any warranty, express or implied, or assumes any legal liability or responsibility for the accuracy, completeness, or any third party's use or the results of such use of any information, apparatus, product, or process disclosed, or represents that its use would not infringe privately owned rights. Reference herein to any specific commercial product, process, or service by trade name, trademark, manufacturer, or otherwise, does not necessarily constitute or imply its endorsement, recommendation, or favoring by the United States Government or any agency thereof or its contractors or subcontractors. The views and opinions of authors expressed herein do not necessarily state or reflect those of the United States Government or any agency thereof. 


\title{
Nm-scale spatial resolution x-ray imaging with MLL nanofocusing optics: instrumentational requirements and challenges
}

\author{
E. Nazaretski ${ }^{1, \text { a) }}$, H. Yan ${ }^{1}$ K. Lauer ${ }^{1}$, X. Huang ${ }^{1}$, W. Xu ${ }^{1}$, S. Kalbfleisch ${ }^{1}$, Hui \\ $\mathrm{Yan}^{1}$, Li Li ${ }^{1}$, N. Bouet ${ }^{1}$, J. Zhou ${ }^{1}$, D. Shu' ${ }^{2}$, R. Conley ${ }^{2}$, and Y. S. Chu ${ }^{1}$ \\ ${ }^{1}$ NSLS-II, Brookhaven National Laboratory, Upton, NY, USA. \\ ${ }^{2}$ Advanced Photon Source, Argonne National Laboratory, Argonne, USA \\ ${ }^{a)}$ Corresponding author: enazaretski@bnl.gov
}

\begin{abstract}
The Hard X-ray Nanoprobe (HXN) beamline at NSLS-II has been designed and constructed to enable imaging experiments with unprecedented spatial resolution and detection sensitivity. The HXN X-ray Microscope is a key instrument for the beamline, providing a suite of experimental capabilities which includes scanning fluorescence, diffraction, differential phase contrast and ptychography utilizing Multilayer Laue Lenses (MLL) and zoneplate (ZP) as nanofocusing optics. We present technical requirements for the MLL-based scanning microscope, outline the development concept and present first $\sim 15 \times 15 \mathrm{~nm}^{2}$ spatial resolution $\mathrm{x}$-ray fluorescence images.
\end{abstract}

Scanning hard x-ray microscopy offers a unique possibility to image samples at a variety of length scales. It provides insight into spatial distribution of components and constituents yielding information regarding chemical composition, dopants, impurities and defects. In recent years a number of scanning hard x-ray microscopes have been designed, constructed and commissioned throughout the world [1-4]. In scanning hard x-ray microscopy, a sample is raster scanned with respect to the incident focused beam and spatial resolution (except ptychography) is determined by properties of nanofocusing optics and stability of the microscope itself. Different nanofocusing optics can be utilized to achieve high spatial resolution in the hard x-ray regime e.g. Kirkpatrick-Baez (KB) mirrors [5], Fresnel Zone Plates [6,7] and Multilayer Laue Lenses [8-11] The Hard X-ray Nanoprobe (HXN) beamline at NSLS-II has been designed and constructed to enable scanning imaging experiments with unprecedented spatial resolution and detection sensitivity. We utilized MLL optics to achieve sub-20 nm focused spot size preserving focusing efficiency in the energy range between 6 and $25 \mathrm{keV}$. A MLL is a linear diffractive optic which overcomes the limited aspect ratio achievable for zone plates using thin film deposition technique. [12,6] In order to achieve point focusing a pair of linear MLLs has to be aligned to satisfy Bragg condition. Moreover the position of both MLL lenses has to remain constant throughout scanning experiments to avoid distortion of images due to possible drifts. When fully commissioned, the HXN microscope will enable 2D fluorescence imaging, 3D tomography, ptychography and diffraction measurements using MLL for high resolution and ZP for moderate resolution experiments. Within this manuscript we focus on the MLL module of the HXN endstation since 2D fluorescence capability has been already commissioned. Figure 1a schematically illustrates layout of the scanning MLL microscope. To perform full alignment of MLL optics five translational and three rotational degrees of motion are required. In addition, an Order Sorting Aperture (OSA) needs to be moved in and out between optics and the sample. For 2D XRF measurements three coarse and fine scanning motions are required at the sample location. For 3D tomography measurements, an additional rotational degree of freedom is needed. Table 1 lists characteristics of motions adequate to perform sub-20 nm imaging experiments. These actuators are already implemented in the HXN 
MLL microscope. Figure 1b-1c are the photographs of the actual MLL module built and installed inside the vacuum chamber of the HXN microscope.

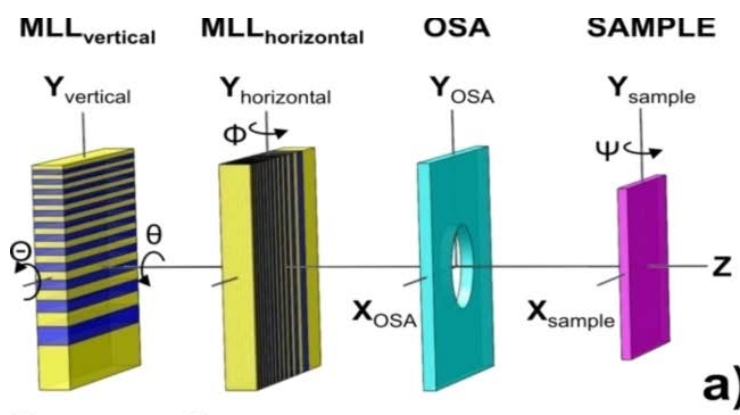

a)

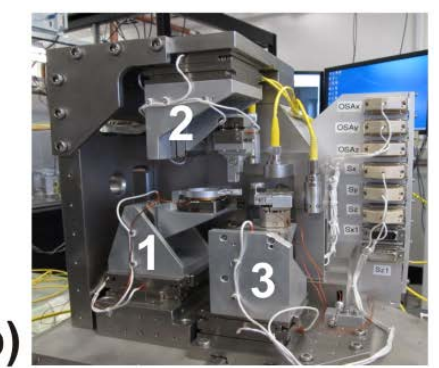

$\mathbf{X}_{\text {vertical }}$

$\mathbf{X}_{\text {horizontal }}$
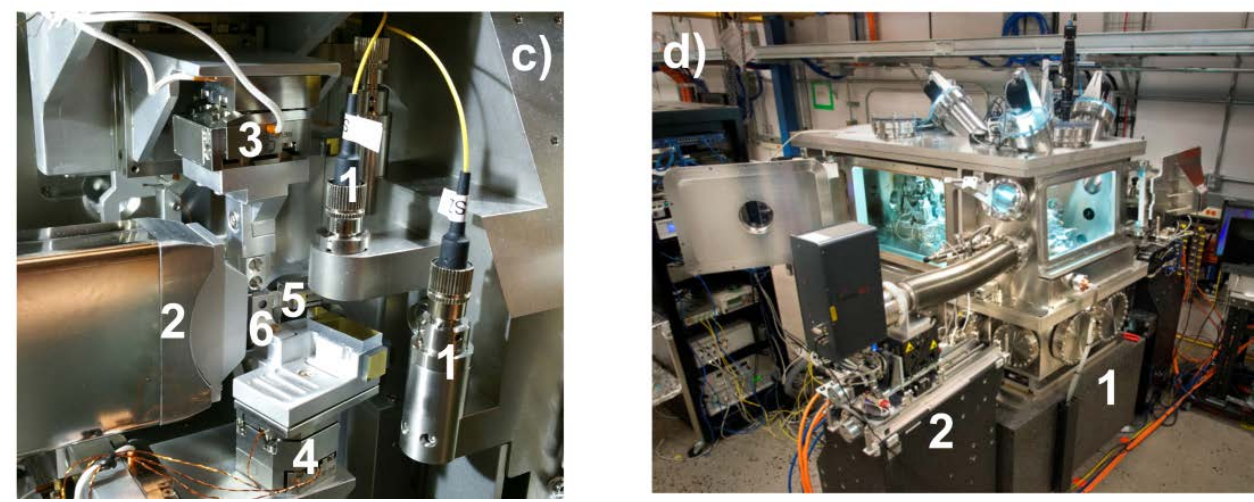

FIGURE 1. a) Schematic layout of an MLL scanning microscope. Major components and corresponding degrees of freedom required to perform full alignment and scanning experiments are illustrated. In total, 5 translational and 3 rotational motions are

needed for alignment of MLL optics. b) Photograph of an MLL module, 1- horizontal MLL assembly, 2 - vertical MLL

assembly, 3 - sample stage. c) detailed view of the sample stage; 1 - interferometers used for feedback and drift corrections, 2 three-element XRF detector, 3 - horizontal MLL assembly, 4 - sample scanner, 5 - OSA, 6 - sample mount. d) Overall view of the microscope with access doors open, 1 - microscope chamber mounted on top of a granite base, 2 - XRF detector on top of manipulation system and granite support.

Prior to construction, rigorous R\&D work has been performed for concept validation and optimization of various components [6, 9-12]. The microscope relies on piezo driven actuators to minimize heat dissipation and at the same time preserve travel ranges and adequate resolution. Their compact size allows to fit the required movements within a small envelope determined by the working distance of MLL optics (for example, $0.8 \mathrm{~mm}$ distance from OSA to the sample at $12 \mathrm{keV}$ energy); nine fiber-optic interferometers monitor the position of both optics and the sample to provide feedback and compensate for possible drifts, some of interferometer heads are shown in panel c) of Fig. 1 and are located in close proximity to monitored components. The interferometer heads are mounted on a common invar reference frame to provide maximum stability. The concept of the microscope echoes approaches used in scanned probe microscopy where all moving components have reduced size to provide maximum stiffness and increase resonance frequencies [13].

TABLE 1. Travel ranges, linear and angular resolutions of piezo motors implemented in the HXN MLL microscope.

\begin{tabular}{ccc}
\hline Name of motion & Travel range & Resolution \\
\hline Vertical MLL (linear) & $20 \mathrm{~mm}$ & $1 \mathrm{~nm}$ \\
Vertical MLL (angular) & $8^{\circ}$ & $1 \times 10^{-4}$ degree \\
\hline
\end{tabular}


Horizontal MLL (linear)

Horizontal MLL (angular)

OSA (linear)

Sample (linear)

Sample fine (scan range)

Sample (angular)

$\begin{array}{cc}20 \mathrm{~mm} & 1 \mathrm{~nm} \\ 8^{\circ} & 1 \times 10^{-4} \text { degree } \\ 20 \mathrm{~mm} & 5 \mathrm{~nm} \\ 20 \mathrm{~mm} & 1 \mathrm{~nm} \\ 40 \mu \mathrm{m} & \text { sub-nm } \\ 180^{\circ} & 1 \times 10^{-4} \text { degree }\end{array}$

A three-element Vortex XRF detector was used for fluorescence mapping in combination with the MLL module. The detector is mounted on a separate granite support and motorized with a large ( $\sim 500 \mathrm{~mm})$ stroke to simplify the sample exchange process or optimize position when collecting data, see panel d) in Fig. 1. First XRF data acquired during commissioning are shown in panel b) of Fig.2. panel a) in the same figure shows the SEM image of a test pattern as a reference.
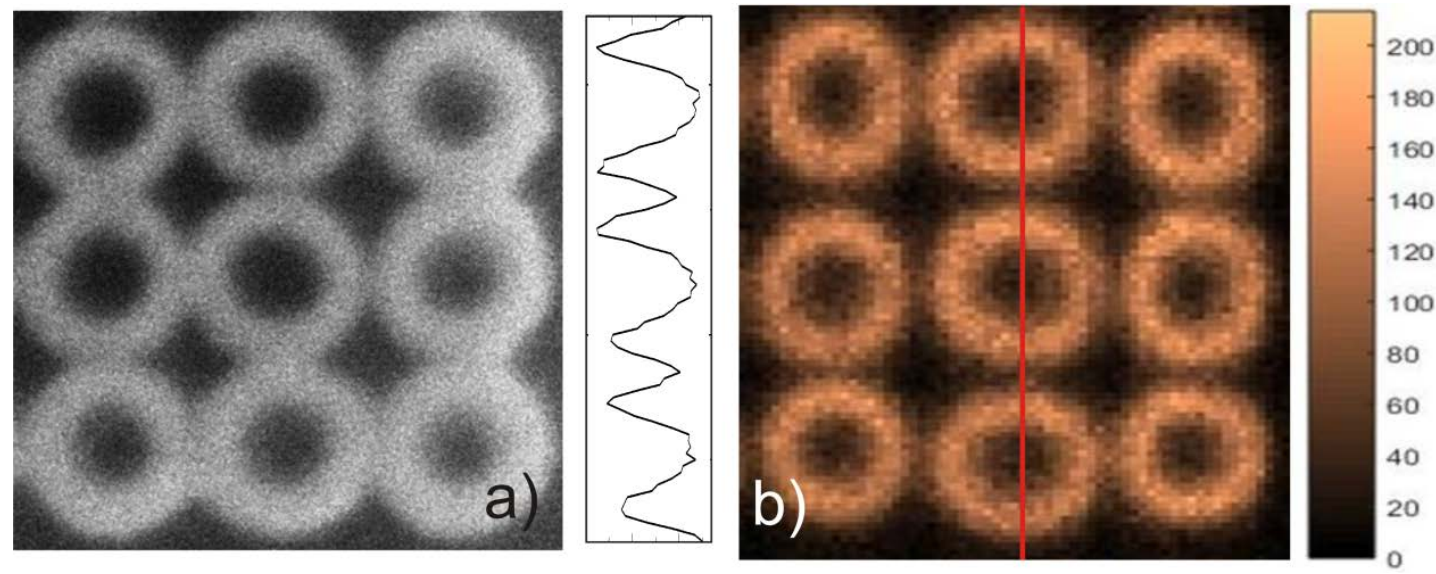

FIGURE 2. Left panel: SEM image of the Pt test pattern. Circles are $80 \mathrm{~nm}$ in diameter and $20 \mathrm{~nm}$ line width. The height of the rings is $200 \mathrm{~nm}$. Right panel: XRF image (platinum L-edge) of the same test pattern, $\mathrm{x}$-ray energy $12 \mathrm{keV}$, exposure time $0.2 \mathrm{~s}, 5$ nm per pixel. Scan profile along horizontal line shown on the left demonstrates achieved spatial resolution.

The test pattern is a set of $80 \mathrm{~nm}$ diameter, $20 \mathrm{~nm}$ wide and $200 \mathrm{~nm}$ high Pt rings, nanofabricated on a thin Si substrate. Imaging measurements were performed using Pt L-line emission with the incidence x-ray energy of 12 $\mathrm{keV}$ and with the synchrotron current of $50 \mathrm{~mA}$. Fly-scan was implemented for data collection with $5 \mathrm{~nm}$ per pixel and 0.2 s exposure time. As seen in Fig 2b, all structures are clearly distinguished and separation between the rings is observed. The resolution measured in both vertical and horizontal directions varies between 13 and 15 nm. As a next step, ptychography, tomography and diffraction capabilities will be commissioned for the MLL module of the HXN microscope.

\section{ACKNOWLEDGMENTS}

Use of the National Synchrotron Light Source II, Brookhaven National Laboratory, was supported by the U.S. Department of Energy, Office of Science, Office of Basic Energy Sciences, under Contract No. DE-SC0012704. Fabrication of test pattern and MLL post-growth processing was performed in part at the Center for Functional Nanomaterials, Brookhaven National Laboratory, supported by the U.S. Department of Energy, Office of Basic Energy Sciences, under Contract No. DE-AC02-98CH10886. 


\section{REFERENCES}

1. R. P. Winarski, M. V. Holt, V. Rose, P. Fuesz, D. Carbaugh, C. Benson, D. Shu, et al., J. Synchrotron Radiation 19, 1056-1060, (2012).

2. Andrea Somogyi, Kadda Medjoubi, Gil Baranton, Vincent Le Roux, Marc Ribbens, Francois Polack, Pascal Philippot and Jean-Pierre Samama, J. Synchrotron Radiation 22, 1118-1129, (2015).

3. S. Chen, J. Deng, Y. Yuan, C. Flachenecker, R. Mak, B. Homberger, Q. Jin, et al., J. Synchrotron Radiation 21, 66-75, (2014).

4. B. Laforce, S. Schmitz, B. Vekemans, J. Rudloff, J. Garrevoet, R. Tucoulou, F. E. Brenker, G. MartinezCriado, and L. Vincze, Anal. Chem. 86, 12369-12374, (2014).

5. $\quad$ K. Yamauchi, H. Mimura, T. Kimura, et al., J. Phys: Condensed Matter 23, 394206 (2011).

6. J. Vila-Comamala, S. Gorelick, E. Farm, C. M. Kewish, A. Diaz, R. Barrett, V. A. Guzenko, M. Ritala and C. David, Opt. Express 19 175-184, (2011)

7. H. R. Wu, S. T. Chen, Y. S. Chu, R. Conley, N. Bouet,C. C. Chien, H. H. Chen, C. H. Lin, H. T. Tung, Y. S. Chen, G. Margaritondo, J. H. Je and Y. Hwu, J. Phys. D: Appl. Phys. 45 242001, (2012).

8. Xiaojing Huang, Hanfei Yan, Evgeny Nazaretski, Raymond Conley et al., Sci. Rep. 3, 3562 (2013).

9. E. Nazaretski, Jungdae Kim, H. Yan, K. Lauer et al., Rev. Sci. Instr. 84, 33701 (2013).

10. E. Nazaretski, K. Lauer, H. Yan, et al. J. Synchrotron Radiation 22, 336-341, (2015).

11. H. Yan, E. Nazaretski, K. Lauer, X. Huang, U. Wagner, C. Rau, Yusuf, I. Robinson, S. Kalblfleisch, L. Li, N. Bouet, J. Zhou, R. Conley, and Y. S. Chu, Scientific Reports, 6, 20112, (2016)

12. W. Chao, B. D. Harteneck, J. A. Liddle, E. H. Anderson and A. D. Attwood, Nature 435 1210-1213, (2005).

13. Jungdae Kim, K. Lauer, H. Yan, Y. S. Chu and E. Nazaretski, Rev. Sci. Instr. 84, 35006 (2013).

14. En-Te Hwu, E. Nazaretski, Y. S. Chu et al., Rev. Sci. Instr. 84, 123702 (2013).

15. E. Nazaretski, X. Huan, H. Yan, K. Lauer et al., Rev. Sci. Instr. 85, 33707 (2014).

16. Weihe Xu, K. Lauer, Y. S. Chu et al., J. Synchrotron Radiation 21, 1367-1369, (2015).

17. E. Nazaretski, K.S. Graham, J. D. Thompson, J. A. Wright et al., Rev. Sci. Instr. 80, 83704 (2009). 